\title{
ON THE WEIERSTRASS PREPARATION THEOREM
}

\section{MATTHEW O'MALLEY}

Introduction. Suppose that $R$ is a commutative ring with identity, $X$ is an indeterminate over $R$, and $S=R[[X]]$ is the formal power series ring. In [1, 33 , Proposition 6], the following result (Weierstrass Preparation Theorem) is proved when $R$ is a local ring, complete in its maximal ideal adic topology: Suppose that $f=\sum_{i=0} a_{i} X^{i} \in S$, where, for some $n \geqq 1, a_{n}$ is a unit of $R$ and $\left(a_{0}, a_{1}, \cdots, a_{n-1}\right) \subseteq M$, the maximal ideal of $R$. Then there exists a unique pair $u, F \in S$ such that $u$ is a unit of $S$ and $F$ is a monic polynomial of degree $n$ with the property that the coefficients of $X^{i}$ in $F$, for $i<n$, are elements of $M$, and such that $f=u F$.

In this paper we extend this result, together with Proposition 5 of $[1, \S 3]$ and its Corollary, to the case when $R$ is any commutative ring with identity and $f=\sum_{i=0} a_{i} X^{i}$ satisfies the property that, for some $n \geqq 1, a_{n}$ is a unit of $R$, while the ideal $A=\left(a_{0}, a_{1}, \cdots, a_{n-1}\right)$ generates a complete Hausdorff topology on $R$.

In $\$ 1$ we give the notation and terminology used throughout the paper, and we prove three results needed in $\$ 2$. $\$ 2$ contains our main results.

All rings considered in this paper are assumed to be commutative and to contain an identity element. The symbols $\omega$ and $\omega_{0}$ are used throughout the paper to denote the sets of positive and nonnegative integers, respectively. A collection of ideals $\left\{A_{k}\right\}_{i k} \in \omega$ of the ring $R$ will be called a $d$-sequence provided that for any $n, m \in \omega$ there exists a $u \in \omega$, depending on $n$ and $m$, such that $A_{u} \subseteq A_{n} \cap A_{m}$.

1. Preliminaries. Let $R$ be a ring, and let $\Omega$ be the topology induced on $R$ by the $d$-sequence $\left\{A_{k}\right\}_{k \in \omega}$ of $R$. We write $(R, \Omega)$ to denote the topological ring $R$ under the topology $\Omega$. It is well known that $(R, \Omega)$ is Hausdorff if and only if $\bigcap_{k \in \omega} A_{k}=(0)$. We say that $(R, \Omega)$ is complete if each Cauchy sequence of $R$ converges to a point of $R$. If there exists an ideal $M$ of $R$ such that $M^{k}=A_{k}$ for each $k \in \omega$, then the topology $\Omega$ is called the $M$-adic topology, and we write $(R, M)$ instead of $(R, \Omega)$ in this case.

(1.1) Lemma. Let $R$ be a ring and suppose that the topology $\Omega$ induced on $R$ by the d-sequence $\left\{A_{k}\right\}_{k \in \omega}$ of $R$ is Hausdorff. Then,

Received by the editors December 9, 1970.

AMS 1970 subject classifications. Primary 13J05, 13J10; Secondary 32B05.

Copyright $\odot 1972$ Rocky Mountain Mathematics Consortium 
if $c \in R$ and if each Cauchy sequence of $(R,(c))$ converges in $(R, \Omega)$, then $(R,(c))$ is a complete Hausdorff space. ((c) denotes the ideal of $R$ generated by $c$.) Moreover, $c$ belongs to the Jacobson radical of $R$.

Proof. Let $c \in R$, and suppose that $(R,(c))$ satisfies the hypothesis of the lemma. Then the proof of Theorem (4.2) of [2] shows that for any $\beta \in S=R[[X]]$ with constant term $c$, there exists an $R$ endomorphism $\phi$ of $S$ mapping $X$ onto $\beta$. But, since $(R, \Omega)$ is Hausdorff, $(R,(c))$ is Hausdorff, and, therefore, by [2, Theorem (4.10)], $(R,(c))$ is complete. Moreover, by [2, Lemma (5.1)], $c$ belongs to the Jacobson radical of $R$.

As a special case of Lemma (1.1), we observe that if $\Omega$ is the $A$-adic topology for some ideal $A$ of $R$, then, for any $c \in A,(R,(c))$ is a complete Hausdorff space if $R$ is complete and Hausdorff in the A-adic topology. Furthermore, $A$ is contained in the Jacobson radical of $R$.

Our next result relates a topological property of $R$ to that of $S$. Namely, if $A$ is an ideal of the ring $R$, then $\left\{A^{k}[[X]]\right\}_{k \in \omega}$ is a $d$ sequence of ideals of $S$, and hence induces a topology $\Lambda$ on $S$. $\left(A^{k}[[X]]\right.$ denotes the set of all power series in $S$ all of whose coefficients are elements of the ideal $A^{k}$ of $R$.) We show that $R$ is complete in the $A$-adic topology if and only if $S$ is complete under the topology $\Lambda$. Since $\bigcap_{k \in \omega}(A)^{k}=(0)$ if and only if $\bigcap_{k \in \omega}\left(A^{k}[[X]]\right)=$ $(0)$, it will follow that $(R, A)$ is a complete Hausdorff space if and only if $(S, \Lambda)$ is a complete Hausdorff space.

(1.2) Lemma. Let $A$ be an ideal of the ring $R$. Then $(R, A)$ is complete if and only if $(\mathrm{S}, \Lambda)$ is complete.

Proof. Suppose that $(S, \Lambda)$ is complete and let $\left\{c_{n}\right\}_{n \in \omega}$ be a Cauchy sequence of $(R, A)$. Since $A^{k} \subseteq A^{k}[[X]]$ for each $k \in \omega$, it follows that $\left\{c_{n}\right\}_{n \in \omega}$ is Cauchy in $(\mathrm{S}, \Lambda)$ and hence, there exists $g=\sum_{i=0}^{\infty} a_{i} X^{i}$ $\in S$ such that $c_{n} \rightarrow g$ in $(S, \Lambda)$. But clearly this implies that $c_{n} \rightarrow a_{0}$ in $(R, A)$. Consequently, $(R, A)$ is complete.

The converse follows from the proof of Lemma (4.6) of [2] .

We note that for any ideal $A$ of the ring $R, A^{n} S=(A S)^{n}$ for each $n \in \omega$, and, if $A$ is finitely generated, then $A^{n} S=A^{n}[[X]]$. Hence, if $A$ is finitely generated, then $\Lambda$ is the $A S$-adic topology on $S$, and Lemma (1.2) shows that $R$ is complete in the $A$-adic topology if and only if $S$ is complete in the AS-adic topology.

We conclude this section with a result needed in the proof of Theorem (2.6). The proof is straightforward and we omit it.

(1.3) Lemma. Let $\beta=\sum_{i=0}^{\infty} a_{i} X^{i} \in S$ and let $A$ denote the ideal $\bigcap_{k \in \omega}\left(a_{0}\right)^{k}$ of $R$. Then $\bigcap_{k \in \omega}(\boldsymbol{\beta})^{k} \subseteq A[[X]]$, where $(\boldsymbol{\beta})$ denotes the 
ideal of $\mathrm{S}$ generated by $\beta$. Therefore, if $\left(R,\left(a_{0}\right)\right)$ is Hausdorff, then $(\mathrm{S},(\boldsymbol{\beta}))$ is Hausdorff.

2. The Weierstrass Preparation Theorem. In this section we give our main results. The proof of our first theorem follows closely the proof of Proposition 5 of $[1$, p. 38], and we only sketch the proof here.

(2.1) Theonem. Let $R$ be a ring with identity and let $f=\sum_{i=0}^{\infty} a_{i} X^{i}$ $\in S$. Suppose that, for some $n \geqq 1, a_{n}$ is a unit of $R$, and that the ideal $A=\left(a_{0}, a_{1}, \cdots, a_{n-1}\right)$ of $R$ generates a complete Hausdorff topology on $R$. If $M$ is the R-submodule of $S$ generated by $\left\{1, X, \cdots, X^{n-1}\right\}$, then $S$ is the direct sum of $M$ and $S$.

Proof. (i) We first observe that $f S \cap M=(0)$. For if

$$
\left(\sum_{i=0}^{\infty} b_{i} X^{i}\right) \cdot f=r_{0}+r_{1} X+\cdots+r_{n-1} X^{n-1},
$$

where $b_{i}, r_{i} \in R$ for each $i$, then the proof of Proposition 5 given in $\left[1\right.$, p. 38] shows that $b_{i} \in \bigcap_{k \in \omega}(A)^{k}$ for each $i \in \omega_{0}$. Hence, since $(R, A)$ is Hausdorff, $b_{i}$ (and, therefore, $r_{i}$ ) is zero for each $i$. Thus, $f S \cap M=(0)$. It should be noted that the proof of (i) depends only on the conditions that $(R, A)$ is Hausdorff and that $a_{n}$ is a unit of $R$.

(ii) We show that $S=f S+M$. If $g=\sum_{i=n}^{\infty} a_{i} X^{i-n}$, then $g$ is a unit of $S$, and $f-X^{n} g=\sum_{i=0}^{n-1} a_{i} X^{i}$. Moreover, if $-h=-\sum_{i=0}^{\infty} h_{i} X^{i}$ $=\left(f-X^{n} g\right) g^{-1}$, then $h_{i} \in A$ for each $i \in \omega_{0}$.

Let $\alpha \in S$. By recursion on $j$, we define a set of elements $q^{(j)}$ of $S$ in the following way:

Let $q^{(0)}$ be the unique element of $S$ satisfying

$$
\boldsymbol{\alpha} \equiv X^{n} q^{(0)}(\bmod M) \text {. }
$$

For $j \in \omega_{0}$, let $q^{(j)}=\sum_{i=0}^{\infty} q_{i}{ }^{(j)} X^{i}$, where, for $j \geqq 1$,

$$
q_{i}^{(j)}=\sum_{k=0}^{i+n} h_{k} q_{i+n-k}^{(j-1)} .
$$

It follows that, for $j \geqq 1$,

$$
X^{n} q^{(j)} \equiv h q^{(j-1)}(\bmod M) .
$$

Since $h_{i} \in A$ for each $i$, it follows from (2.3), by induction on $n$, that $q_{i}^{(j)} \in A^{j}$ for all $i \in \omega_{0}$ and each $j \in \omega$. It follows, therefore, that $\left\{\sum_{j=0}^{t} q^{(j)}\right\}_{t \in \omega_{0}}$ is a Cauchy sequence of $S$ in the topology $\Lambda$ induced on $S$ by the sequence of ideals $\left\{A^{k}[[X]]\right\}_{k \in \omega^{*}}$ Thus, since $(R, A)$ is a complete Hausdorff space, it follows from Lemma (1.2) that 
$(S, \Lambda)$ is a complete Hausdorff space. Therefore, there exists a unique element $q \in S$ such that $q$ is the limit of the sequence $\left\{\sum_{j=0}^{t} q^{(j)}\right\}_{t \in \omega_{0}}$ in $(S, \Lambda)$. From (2.2) and (2.4) we have that

$$
\alpha+h\left(\sum_{j=0}^{t-1} q^{(j)}\right) \equiv X^{n}\left(\sum_{j=0}^{t} q^{(j)}\right)(\bmod M),
$$

for each $t \in \omega$, and hence

$$
\alpha+h\left(\sum_{j=0}^{t-1} q^{(j)}\right)-X^{n}\left(\sum_{j=0}^{t} q^{(j)}\right)=m_{t} \in M,
$$

for each $t \in \omega$. Thus, since the limit on the left exists in $(S, \Lambda)$, the limit on the right exists, and we have that

$$
\alpha+h q-X^{n} q=\lim _{t} m_{t}
$$

Therefore,

$$
\begin{aligned}
\alpha & =\left(X^{n}-h\right) q+\lim _{t} m_{t} \\
& =f g^{-1} q+\lim _{t} m_{t},
\end{aligned}
$$

where $f g^{-1} q \in f S$. Thus, it suffices to show that $M$ is closed in $(S, \Lambda)$. But this is straightforward and we omit it.

Note that the proof of the equality of $f S \cap M=(0)$ shows that $f$ is regular (not a zero divisor) in $S$. From this fact, it follows that if $\alpha=h f+\sum_{i=0}^{n-1} r_{i} X^{i}=h_{1} f+\sum_{i=0}^{n-1} u_{i} X^{i}$ are two representations for $\alpha$ as an element of $f S+M$, then $h=h_{1}$ and $r_{i}=u_{i}$ for $0 \leqq i \leqq n-1$.

If $g=\sum_{i=0}^{\infty} c_{i} X^{i} \in S$, where $R$ is complete and Hausdorff in the $\left(c_{0}\right)$-adic topology, then [2, Theorems (4.2) and (4.3)] shows that there exists a unique $R$-endomorphism $\phi_{g}$ of $S$ that maps $X$ onto $g$. We denote the range of $\phi_{g}$ by $R[[g]]$.

In particular, if $f=\sum_{i=0}^{\infty} a_{i} X^{i} \in S$, and if $f$ satisfies the hypothesis of Theorem (2.1), then it follows from Lemma (1.1) that $R$ is complete and Hausdorff in the $\left(a_{0}\right)$-adic topology. Thus, there exists a unique $R$-endomorphism $\phi_{f}$ of $S$ such that $\phi_{f}(X)=f$. We next show that, for any $f \in S$ satisfying the hypothesis of Theorem (2.1), $\phi_{f}$ is one-toone. We make use of [3] to prove a more general result than this.

(2.5) Lemma. Let $g \in S$ and suppose that there exists an $R$ endomorphism $\psi$ of $S$ mapping $X$ onto $g$. If $T$ denotes the range of $\psi$, and if $g$ satisfies the following conditions:

(i) g is regular in $T$, and

(ii) $g T \cap R=(0)$, 
then $\psi$ is one-to-one. In particular, if $f$ satisfies the hypothesis of Theorem (2.1), then $\phi_{f}$ is one-to-one.

Proof. Let $h=\sum_{j=0}^{\infty} h_{j} X^{j} \in S$ and suppose that $\psi(h)=0$. By [3, Result (2.1)], $\psi(h)$ is a limit point in $(T,(g T))$ of the sequence $\left\{\sum_{j=0}^{n} h_{j} g^{j}\right\}_{n} \in \omega_{0}$.

Fix $k \in \omega$. We show that $h_{j}=0$ for $0 \leqq j \leqq k$. Note that $T$ contains $R[g]$, the subring of $S$ consisting of all elements of the form $\sum_{i=0}^{u} r_{i} g^{i}, r_{i} \in R$. Now, since $\psi(h)=0$, we have that $\sum_{j=0}^{n} h_{j} g^{j} \rightarrow 0$ in $(T,(g T))$, and therefore, for $\left(g^{k+1} T\right)$, a neighborhood of 0 , there exists $N \in \omega, N>k$, such that $\sum_{j=0}^{n} h_{j} g^{j} \in\left(g^{k+1} T\right)$ for $n \geqq N$. Let $\sum_{j=0}^{n} h_{j} g^{j}=g^{k+1} \alpha, \alpha \in T$. Then,

$$
h_{0}=-g\left(\sum_{j=1}^{n} h_{j} g^{j-1}-g^{k} \boldsymbol{\alpha}\right),
$$

and, hence, by (ii), $h_{0}=0$.

Suppose we have shown that $h_{j}=0$ for $0 \leqq j<r \leqq k$. We show that $h_{r}=0$. By the induction hypothesis, we have $\sum_{j=r}^{n} h_{j} g^{j}=g^{k+1} \alpha$, and thus, $g^{r}\left(\sum_{j=r}^{n} h_{j} g^{j-r}-g^{k+1-r} \alpha\right)=g^{r} u=0$. Since $u \in T$, it follows from (i) that $u=\sum_{j=r}^{n} h_{j} g^{j-r}-g^{k+1-r} \alpha=0$. Thus

$$
h_{r}=-g\left(\sum_{j=r+1}^{n} h_{j} g^{j-(r+1)}-g^{k-r} \boldsymbol{\alpha}\right),
$$

and therefore, $h_{r}=0$. It follows, by induction, that $h_{j}=0$ for $0 \leqq j \leqq k$, and thus, since $k$ was arbitrary, we have that $h=0$. This completes the proof.

Our next result corresponds to the Corollary to Proposition 5 of [ 1 , p. 40].

(2.6) THEOREM. Let $f \in S$ and suppose that $f$ satisfies the hypothesis of Theorem (2.1). Then $\left\{1, X, \cdots, X^{n-1}\right\}$ is a free-module basis for $S$ over $R[[f]]$. Furthermore, the unique R-endomorphism $\phi_{f}$ of $\mathrm{S}$ mapping $\mathrm{X}$ onto fis one-to-one.

Proof. We have already observed the last statement of the theorem (Lemma (2.5)). We prove the first part of the theorem in a series of steps.

For each $k \in \omega$, let $T_{k}$ denote $f^{k-1} R+f^{k-2} R+\cdots+f R+R$, the $R$-submodule of $R[f]$ consisting of all terms of the form: $\sum_{i=0}^{k-1} r_{i} f^{i}, r_{i} \in R$. (Note that $T_{1}=R$.) We first observe that, for any $k \in \omega$,

$$
S=f^{k} S+T_{k} \cdot 1+T_{k} \cdot X+\cdots+T_{k} \cdot X^{n-1} .
$$


The case for $k=1$ is proved in Theorem (2.1), and, for $k>1$, the proof follows easily by induction on $k$.

We next observe that, for any $k \in \omega$,

$$
f^{k} S \cap\left[T_{k} \cdot 1+T_{k} \cdot X+\cdots+T_{k} \cdot X^{n-1}\right]=(0) .
$$

Again the case for $k=1$ is proved in Theorem (2.1). We suppose that (2.8) is valid for $k=u$, and we let

$$
g f^{u+1}=\sum_{j=0}^{n-1}\left(\sum_{i=0}^{u} r_{i}^{(j)} f^{i}\right) X^{j},
$$

where $g \in S, r_{i}^{(j)} \in R$ for all $i, j$. It follows that

$$
g f^{u+1}=\left(\sum_{j=0}^{n-1} r_{u}^{(j)} X^{j}\right) f^{u}+\sum_{j=0}^{n-1}\left(\sum_{i=0}^{u-1} r_{i}^{(j)} f^{i}\right) X^{j},
$$

and hence,

$$
\left[g f-\sum_{j=0}^{n-1} r_{u}^{(j)} X^{j}\right] f^{u}=\sum_{j=0}^{n-1}\left(\sum_{i=0}^{u-1} r_{i}^{(j)} f^{i}\right) X^{j} .
$$

Therefore, by the induction hypothesis and since $f$ is regular in $S$, we have that

$$
g f-\sum_{j=0}^{n-1} r_{u}^{(j)} X^{j}=0
$$

and

$$
\sum_{j=0}^{n-1}\left(\sum_{i=0}^{u-1} r_{i}^{(j)} f^{i}\right) X^{j}=0 .
$$

In particular, since $g f=\sum_{j=0}^{n-1} r_{u}{ }^{(j)} X^{j}$, it follows from the case for $k=1$ that $g=0$, and we have proved (2.8). We note that it follows from the proof of (2.8) that if $\sum_{j=0}^{n-1}\left(\sum_{i=0}^{k} r_{i}^{(j)} f^{i}\right) X^{j}=0$ for any $k \in \omega_{0}$, then $r_{i}^{(j)}=0$ for all $i, j$. From this observation and from (2.8), it follows that if $\alpha \in S$ and if

$$
\alpha=f^{k} s_{k}+\sum_{j=0}^{n-1}\left(\sum_{i=0}^{k-1} r_{i}^{(j) f^{i}}\right) X^{j},
$$

and

$$
\alpha=f^{k+r} s_{k+r}+\sum_{j=0}^{n-1}\left(\sum_{i=0}^{r+(k-1)} t_{i}^{(j)} f^{i}\right) X^{j}
$$


are two representations for $\alpha$ as an element of

$$
f^{k} S+T_{k} \cdot 1+T_{k} \cdot X+\cdots+T_{k} \cdot X^{n-1},
$$

and

$$
f^{k+r} S+T_{k+r} \cdot 1+T_{k+r} \cdot X+\cdots+T_{k+r} \cdot X^{n-1},
$$

respectively, then $r_{i}^{(j)}=t_{i}^{(j)}$ for each $j$ and for $0 \leqq i \leqq k-1$. Hence, in our representation for $\alpha$, as $k \rightarrow \infty$, the $r_{i}^{(j)}$ for $i<k$ remain fixed, and, in this sense, are independent of $k$.

Let $\alpha \in S$ and suppose that

$$
\left\{f^{k} s_{k}+\sum_{j=0}^{n-1}\left(\sum_{i=0}^{k-1} r_{i}^{(j)} f^{i}\right) X^{j}\right\}_{k \in \omega}
$$

is the collection of representations of $\alpha$ as an element of the sets $\left\{f^{k} S+T_{k} \cdot 1+T_{k} \cdot X+\cdots+T_{k} \cdot X^{n-1}\right\}_{k \in \omega}$. We show that

$$
\alpha=\sum_{i=0}^{\infty} r_{i}^{(0)} f^{i}+\left(\sum_{i=0}^{\infty} r_{i}^{(1)} f^{i}\right) X+\cdots+\left(\sum_{i=0}^{\infty} r_{i}^{(n-1)} f^{i}\right) X^{n-1}
$$

This will show that

$$
S=R[[f]] \cdot 1+R[[f]] \cdot X+\cdots+R[[f]] \cdot X^{n-1} .
$$

To prove (2.9), we show that the sequence of points

$$
\left\{f^{k} s_{k}+\sum_{j=0}^{n-1}\left(\sum_{i=0}^{k-1} r_{i}^{(j)} f^{i}\right) X^{j}\right\}_{k \in \omega}=\{\alpha\}_{k \in \omega}
$$

of $S$ converges in the $(f)$-adic topology on $S$ to the right-hand side of (2.9). Hence, since $\{\boldsymbol{\alpha}\}_{k \in \omega} \rightarrow \boldsymbol{\alpha}$, and since $S$ is Hausdorff in the (f)-adic topology (Lemma (1.3)), it will follow that (2.9) is true. Let $(f)^{m}$ be a neighborhood of 0 in $(S,(f))$ and choose $k \geqq m$. Then

$$
\begin{aligned}
& \sum_{j=0}^{n-1}\left(\sum_{i=0}^{\infty} r_{i}^{(j)} f^{i}\right) X^{j}-\left(f^{k} s_{k}+\sum_{j=0}^{n-1}\left(\sum_{i=0}^{k-1} r_{i}^{(j)} f^{i}\right) X^{j}\right) \\
& =\sum_{j=0}^{n-1}\left(\sum_{i=k}^{\infty} r_{i}^{(j)} f^{i}\right) X^{j}-f^{k} s_{k} \\
& =f^{k}\left(\left[\sum_{j=0}^{n-1}\left(\sum_{i=0}^{\infty} r_{i+k}^{(j)} f^{i}\right) X^{j}\right]-s_{k}\right) \in(f)^{k} \subseteq(f)^{m}
\end{aligned}
$$


Finally, we observe that arguments similar to those already used in the proof show that $\left\{1, X, \cdots, X^{n-1}\right\}$ is a free basis, and we omit the proof.

REMark. It should be observed here that Theorem (2.6) has an important application in the generalization of a result of Samuel [4]. In particular, let $R$ be a Noetherian integral domain with identity whose integral closure is a finite $R$-module, let $S=R[[X]]$, and let $G=\left\{\phi_{i}\right\}_{i=1}^{n}$ be a finite group of $R$-automorphisms of $S$. If $f=$ $\prod_{i=1}^{n} \phi_{i}(X)=\sum_{i=0}^{\infty} a_{i} X^{i}$, then it can be shown that the coefficients of $f$ satisfy the hypothesis of Theorem (2.1), and hence, $\left\{1, X, \cdots, X^{n-1}\right\}$ is a free-module basis for $S$ over $R[[f]]$. This result is of prime importance in the proof that the ring $S^{G}=\left\{h \in S \mid \phi_{i}(h)=h\right.$ for each $i=1, \cdots, n\}$ of invariants of $G$ is $R[[f]]$. The details will appear elsewhere.

Next, we give our extension of Proposition 6 of $[1$, p. 41] .

(2.10) Theorem. Let $R$ be $a$ ring with identity and let $f=$ $\sum_{i=0}^{\infty} a_{i} X^{i} \in S$. Suppose that for some $n \geqq 1, a_{n}$ is a unit of $R$, and suppose that the ideal $A=\left(a_{0}, a_{1}, \cdots, a_{n-1}\right)$ of $R$ generates a complete Hausdorff topology on $R$. Then there exists a unique pair $u$, $F \in S$ such that $u$ is a unit of $S, F=r_{0}+r_{1} X+\cdots+r_{n-1} X^{n-1}+$ $X^{n}$, where $r_{i} \in A$ for each $i$, and $f=u F$.

Proof. By Theorem (2.1), $X^{n}$ has a unique representation as an element of $f S+M$, where $M$ is the $R$-submodule of $S$ generated by $\left\{1, X, \cdots, X^{n-1}\right\}$. Let

$$
X^{n}=g f+\left(r_{0}+r_{1} X+\cdots+r_{n-1} X^{n-1}\right) .
$$

Then $g f=X^{n}-\left(r_{0}+r_{1} X+\cdots+r_{n-1} X^{n-1}\right)$. Thus, if $g=\sum_{j=0}^{\infty} g_{j} X^{j}$, then the coefficient of $X^{n}$ in $g f$ is $\sum_{i+j=n} a_{i} g_{j}$, and, by definition of equality in $S$, we have that $\sum_{i+j=n} a_{i} g_{j}=1$. Therefore, $a_{n} g_{0}=1-$ $\left(a_{n-1} g_{1}+\cdots+a_{0} g_{n}\right)$, and, since $(R, A)$ is a complete Hausdorff space, it follows from the remark following Lemma (1.1) that $a_{n-1} g_{1}+\cdots+a_{0} g_{n}$ is in the Jacobson radical of $R$. By [5, Lemma 2 , p. 206], it follows that $a_{n} g_{0}$ is a unit of $R$, and therefore $g_{0}$ is a unit of $R$. Thus, $g$ is a unit of $S[6, p .131]$, and $f=$ $g^{-1}\left(X^{n}-r_{n-1} X^{n-1}-\cdots-r_{0}\right)$. Since $r_{k}=\sum_{i+j=k} a_{i} g_{j}$ for $0 \leqq k \leqq n$ -1 , each $r_{k} \in A$. Therefore $u=g^{-1}$ and $F=-r_{0}-r_{1} X-\cdots-$ $r_{n-1} X^{n-1}+X^{n}$ satisfy the conclusion of the theorem, and $f=u F$.

The uniqueness of $u$ and $F$ follows easily from the uniqueness of the representation (2.11), and we omit the details. This completes the proof. 


\section{REFERENCES}

1. N. Bourbaki, Albèbre commutative. Chap. 7: Diviseurs, Actualités Sci. Indust., no. 1314, Hermann, Paris, 1965. MR 41 \#5339.

2. M. O’Malley, R-automorphisms of $R[[X]]$, Proc. London Math. Soc. (3) 20 (1970), 60-78. MR 40 \#7249.

3. M. O'Malley and C. Wood, R-endomorphisms of $R[[X]]$, J. Algebra 15 (1970), 314-327. MR 41 \#8407.

4. P. Samuel, Groupes finis d'automorphismes des anneaux de séries formelles, Bull. Sci. Math. (2) 90 (1966), 97-101. MR 35 \#180.

5. O. Zariski and P. Samuel, Commutative algebra. I, Univ. Series in Higher Math., Van Nostrand, Princeton, N. J., 1958. MR 19, 833.

6. —_ Commutative algebra. II, Univ. Series in Higher Math., Van Nostrand, Princeton, N. J., 1960. MR 22 \#11006.

NaSa Manned Spacecraft Center, Mail Code: ED 3, Houston, Texas 77058 
